\title{
Sub-wavelength phononic crystal liquid sensor
}

\author{
Manzhu Ke, ${ }^{1,2, a)}$ Mikhail Zubtsov, ${ }^{1}$ and Ralf Lucklum ${ }^{1, a)}$ \\ ${ }^{1}$ Institute of Micro and Sensor Systems, Otto-von-Guericke-University, Magdeburg, Germany \\ ${ }^{2}$ Department of Physics, Wuhan University, China
}

(Received 21 April 2011; accepted 3 June 2011; published online 18 July 2011)

\begin{abstract}
We introduce an acoustic liquid sensor based on phononic crystals consisting of steel plate with an array of holes filled with liquid. We both theoretically and experimentally demonstrate sensor properties considering the mechanism of the extraordinary acoustic transmission as underlying phenomenon. The frequency of this resonant transmission peak is shown to rely on the speed of sound of the liquid, and the resonant frequency can be used as a measure of speed of sound and related properties, like concentration of a component in the liquid mixture. The finite-difference time domain method has been applied for sensor design. Ultrasonic transmission experiments are performed. Good consistency of the resonant frequency shift has been found between theoretical results and experiments. The proposed scheme offers a platform for an acoustic liquid sensor. (C) 2011 American Institute of Physics. [doi:10.1063/1.3610391]
\end{abstract}

An ultrasonic broadband measurement of absorption and velocity in liquids and liquid systems has been proven to be an almost universal tool for many applications in basic research as well as in characterization of materials, manufacturing processes, and control routines. ${ }^{1}$ Sound waves in liquids induce perturbations, affecting equilibrium and resulting in characteristic sound absorption and velocity dispersion. Precise attenuation or velocity measurements yield valuable information about thermodynamic and kinetic parameters of the liquid that is often difficult to obtain by other methods. Though these amplitude-based or bulk-resonant measurement techniques are relatively easy in the use at macro-scale, they are rather challenging in applications at micro-scale, in particular, in combination with micro-fluidic devices, because of a short propagation path of ultrasonic waves.

During the last three decades, phononic crystal structures $(\mathrm{PnC})$ have gained much attention due to their rich physics and great utility in the control of sound propagation. The reported advances in PnCs implementation and fabrication promise both their efficient and beneficial integration into existing technologies and far greater experimentation on a wide variety of potential applications. ${ }^{2,3}$

Recently, an extraordinary acoustic transmission (EAT) through a structure with regularly perforated apertures immersed in a liquid has been demonstrated when acoustic waves are incident either on one-dimensional gratings with narrow apertures ${ }^{4}$ or on $2 \mathrm{D}$ perforated metal plates with arrays of holes with sub-wavelength size. ${ }^{5-9}$ There are no generally recognized theory or conceptual framework concerning EAT, though the behavior of acoustic waves propagating through these structures has been a subject of intensive research. Lu et $a l .{ }^{4}$ have attributed the resonant enhancement in EAT to the coupling between the compositions of diffractive waves excited on the surfaces of the gra-

\footnotetext{
a) Authors to whom correspondence should be addressed. Electronic addresses: mzke@whu.edu.cn and ralf.lucklum@ovgu.de.
}

ting and the Fabry-Perot (FP) resonant modes inside the apertures. Hou et al. ${ }^{5,6}$ have demonstrated that evanescent waves may alter FP resonance conditions and FP resonance can occur in aperture with the length, which is even less than the half wavelength of incident wave. They have also found that both the periodicity and area fraction of apertures may affect the FP resonance condition and that this resonance is smoothly linked to the surface-wave-like modes induced by the periodic structure factor. Estrada et al. $^{7}$ point out that there is a number of several transmission mechanisms involved in EAT and producing complex interplay phenomena: transmission assisted by cutoff-free waveguide modes of individual apertures, interaction among holes arranged in array, and direct transmission through the bulk material. Wang $^{9}$ has introduced an acoustic view of this phenomenon, specifically focusing on the properties of the liquid.

In this paper, we demonstrate how extraordinary acoustic transmission in phononic crystals can be used to determine mechanical material properties of liquids. We follow the route of Huang's research paper, ${ }^{10}$ which succeeded in realizing the sub-wavelength nanofluidics in photonic crystal sensors by using the similar resonance transmission properties. They applied this method to detect refractive index changes in aqueous solutions and showed enhancements in the sensor performance and the sensitivity. We have studied a specific arrangement of PnC which is typical for EAT experiments, specifically, a thin plate with the thickness comparative to the half wavelength of the source of sound, regularly perforated with circular holes and immersed in liquid.

We have chosen the size of the structure in millimeter scale range. The structure (shown in Fig. 1(a)) we used here consists of a steel plate with liquid holes in square arrays. The lattice distance (denoting as $a$ ) is $1.5 \mathrm{~mm}$, the thickness $(t)$ of the plate is $0.5 \mathrm{~mm}$ and the diameter $(d)$ of the hole is $0.5 \mathrm{~mm}$. Different liquid material properties are obtained by gradually changing the liquid in the holes and surroundings from pure Distilled water (DI-water) through a series of liquid mixtures with different molar concentration to pure 1-propanol. 

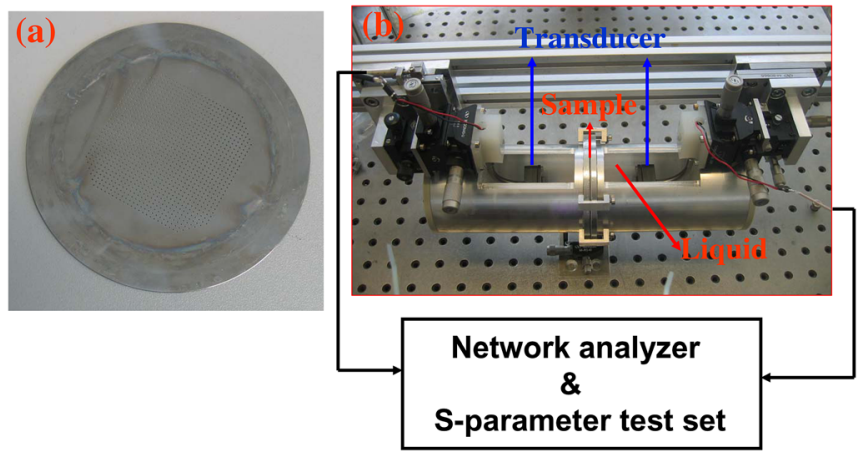

FIG. 1. (Color online) Photo of the sample structure (left) and the experimental setup (right).

We calculate the transmission spectrum by using threedimensional finite-difference time-domain (FDTD) method. ${ }^{11}$ Based on the elastic wave equations in homogeneous medium, assuming the initial stress and the speed of any point is zero, we can get the velocity vector and the stress relation equations. ${ }^{12}$ We have obtained the relation equations of stress and the velocity, which use discretization of the equations in both the space and the time domains. In the FDTD calculations, we choose a computational cell with dimension $30 \times 30 \times 300$ grid points, corresponding to 30 grid points per lattice constant $a$. A spatial grid with mesh size $\Delta x=\Delta y=\Delta z=0.05$ $\mathrm{mm}$ is used. The faces of the computational cell normal to the $x$ and $y$ axes are chosen to have Bloch boundary conditions, while the faces normal to $z$ axis (the top and bottom ones) use Mur's absorbing boundary conditions. For transmission spectrum calculations, a broad band wave packet is launched to illuminate the plate with a normal incidence. The transmitted signal is recorded as a function of time. The transmission coefficient is calculated by dividing the transmitted energy flux by the energy flux of the incident wave.

For the experimental measurements, the transmission curves are obtained using a network analyzer (Agilent 4395 A) together with a S-parameter test set (Agilent $87511 \mathrm{~A}$ (100 kHz-500 MHz)). A small, in-house developed liquid container is used to fix the sample and fill the liquid in the holes of the plate. The sample was placed between two piezoelectric transducers with a central frequency of $1.0 \mathrm{MHz}$, and the distance between the transducers and metal plate was far enough to avoid near field effect and multiple reflections. The illustration of the experimental setup is shown in Fig. 1(b). Transmission S21 has been measured with and without sample in place, respectively. Then, the transmission amplitude was obtained by normalizing with the amplitude of the equivalent setup without the sample in place.

The transmission spectrum of the system filled with water is shown Fig. 2, where the dashed line indicates the experimental measurement and the solid lines denote the theoretical simulation. As predicted, there is an extraordinary transmission peak appearing at the frequency range of $800 \mathrm{kHz}$ to 900 $\mathrm{kHz}$. Evaluating from the shape and the line-widths and transmission maximum amplitude, this resonant peak is suitable for sensor applications. Nearly perfect match between the resonance shape and the line-widths are observed for both experiment and simulation. But there are slight discrepancies

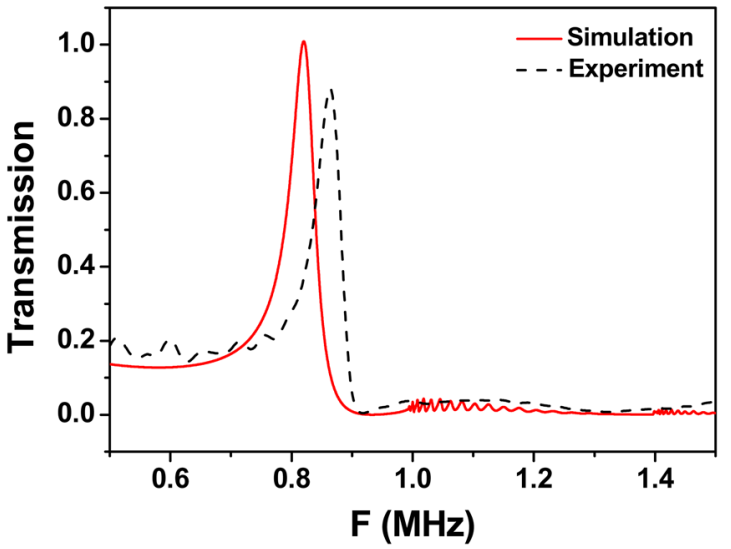

FIG. 2. (Color online) Transmission spectrum of the sensor system with water, where the dashed line indicates the experimental measurement and the solid lines indicate the FDTD simulation results.

in the resonance frequency location and the transmission amplitude, which should be attributed to the experimental limitations. On one hand, the fabrication of the sample is not perfect, the accurate size of the lattice constant and diameter of every hole in the plate and complete symmetry for the sample are difficult to realize across the whole sample; on the other hand, part of the loss and absorption and variation in the signal response with time are inevitably caused by the experimental circumstances, such as the walls of the small container and the impurity of water, etc.

In order to demonstrate the basic characteristics of how the material properties of the liquid effect on the EAT mixtures of DI water and 1-propanol with molar ratios of 0.021 , $0.035,0.056,0.102,0.158,0.230,0.347,0.507$, and 0.596, respectively, have been used. The speed of sound and density of all liquids are known from literature. ${ }^{13}$ Prior to measurement, we have made sure the former solution is entirely replaced by the latter one. Figure 3 gives the frequency responses with molar ratio of 1-propanol. We observed, both in FDTD simulation and in experiment, that the frequency of

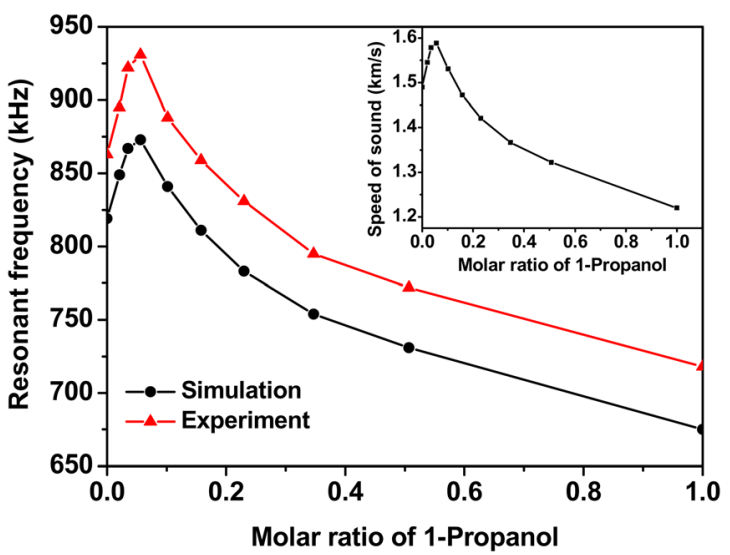

FIG. 3. (Color online) Results of resonant frequency vs molar ratio of 1propanol in the liquid mixtures. The curve with circular symbols shows the FDTD simulation and the curve with triangular symbols denotes the experimental measurement. The maximum frequency position of the resonant transmission peak corresponds to the highest speed of sound at molar ratio $\mathrm{x}_{2}=0.056$. The inset shows the speed of sound vs molar ratio of 1-propanol in the liquid mixtures. 


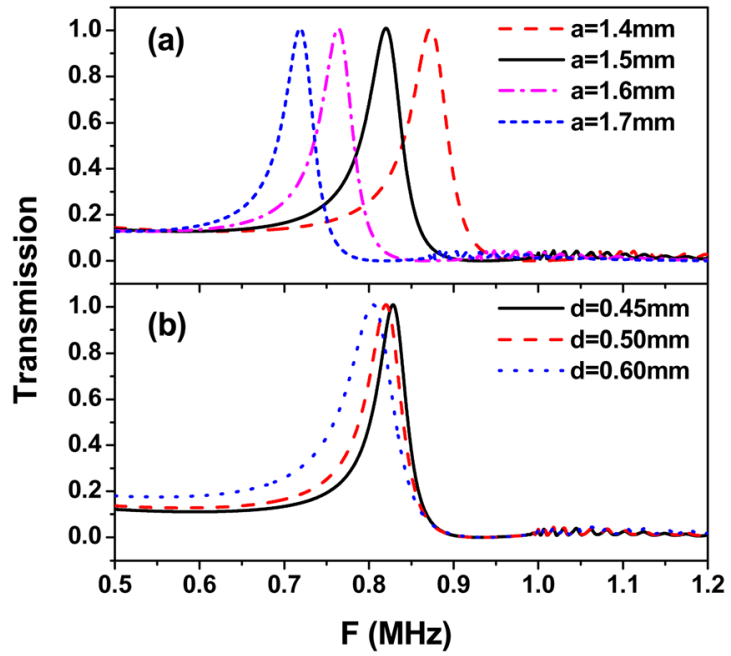

FIG. 4. (Color online) Transmission spectrum of the sensor system with water with different lattice constant (a) and the hole diameter of the plate (b).

resonant transmission peak significantly changes with the molar ratio of 1-propanol in the liquid mixtures. The inset in Fig. 3 gives the speed of sound of the mixtures versus the molar ratio, reflecting almost the same behavior as the frequency response curve. Obviously, the shift of the maximum transmission peak frequency reflects the difference in speed of sound; the highest resonance frequency appears at molar ratio $\mathrm{x}_{2}=0.056$, corresponding to the maximum speed of sound of $v=1588 \mathrm{~m} / \mathrm{s}$ of the liquid mixture. Since the molar ratio of 1-propanol governs the speed of sound of the liquid, the shift of the resonant frequency can serve as a sensor for both measuring the concentration of one component in the mixture and the speed of sound of the liquid. Again, the same as in Fig. 2, there is a systematic error in the frequency between simulation and experiment.

To simply evaluate the sensitivity of the sensor, we calculated $S_{f}$, which can be defined as the ratio of frequency shift $(\Delta f)$ to the change of input parameter $(\Delta x): S_{f}=\frac{\Delta f}{\Delta x}$. Taking the range between $\mathrm{x}_{2}=0$ to 0.035 as an example, the sensitivity $\mathrm{S}_{f}$ can be estimated to be $1370 \mathrm{kHz}$ (simulation) and $1680 \mathrm{kHz}$ (experiment). Taking a conservative estimate of the frequency resolution of $1 \mathrm{kHz}$, the sensor would have a limit of detection of $0.07 \%$ molar ratio in this range. Since the use of a silicon platform paves the way toward miniaturized sensors, this kind of sensor can also be rescaled to smaller wavelength, i.e., giving access to higher sensitivity and smaller analyte volume, which is, for example, important for biosensor applications.

In order to understand the systematic error, Fig. 4 illustrates the result of variation in the lattice constant $a$ from 1.4 $\mathrm{mm}$ to $1.7 \mathrm{~mm}$ and the hole diameter $d$ from $0.45 \mathrm{~mm}$ to $0.60 \mathrm{~mm}$. Compared to the holes diameter, the lattice constants obviously take the main role in cross sensitivity: about
$1 \%$ change of lattice constant leads to $7-8 \mathrm{kHz}$ shift of the transmission peak frequency, while about $1 \%$ changes for diameter of holes only leads to $0.7 \mathrm{kHz}$.

Finally, we have analyzed effects arising from the materials themselves. Longitudinal and transverse components of sound velocity and density of the steel plate have been varied by $1 \%$, resulting in a peak frequency of $0.04 \mathrm{kHz}, 0.19 \mathrm{kHz}$, and $0.12 \mathrm{kHz}$, respectively. Varying shift density of water by $1 \%$ gives rise to a frequency shift of $0.12 \mathrm{kHz}$. The results clearly show that parameters have very minor effect on the resonant peak position compared to speed of sound of the liquid. The above results prove that the extraordinary transmission peak can be used to determine speed of sound of the liquid with a phononic crystal sensor as direct and concentration of a mixture as an indirect value, the latter providing a reasonable relation between concentration and speed of sound.

In summary, our study demonstrates that phenomenon of extraordinary acoustic transmission through a phononic crystal structure can be beneficially utilized for liquid sensor purposes. We have both theoretically and experimentally demonstrated the sensor properties by using the resonant transmission peak as a favorable measure. The position of the peak frequency has been proven to be highly sensitive to the speed of sound of the liquid surrounding the phononic crystal plate and filling its holes. We have also demonstrated that the molar ratio of one component of a liquid mixture can be determined.

\section{ACKNOWLEDGMENTS}

This work has been supported by FET-Open Project TAILPHOX (Grant No. 233883) and the German Research Foundation (Lu 605/12-1).

${ }^{1}$ U. Kaatze, F. Eggers, and K. Lautscham, Meas. Sci. Technol. 19, 062001 (2008).

${ }^{2}$ R. H. Olsson III and I. El-Kady, Meas. Sci. Technol. 20, 012002 (2009).

${ }^{3}$ R. Lucklum and J. Li, Meas. Sci. Technol. 20, 124014 (2009).

${ }^{4}$ M. H. Lu, X. K. Liu, L. Feng, J. Li, C. P. Huang, Y. F. Chen, Y. Y. Zhu, S. N. Zhu, and N. B. Ming, Phys. Rev. Lett. 99, 174301 (2007).

${ }^{5}$ B. Hou, J. Mei, M. Ke, W. Wen, Z. Liu, J. Shi, and P. Sheng, Phys. Rev. B 76, 054303 (2007).

${ }^{6}$ B. Hou, J. Mei, M. Ke, Z. Liu, J. Shi, and W. Wen, J. Appl. Phys. 104, 014909 (2008).

${ }^{7}$ H. Estrada, P. Candelas, A. Uris, F. Belmar, F. J. García de Abajo, and F. Meseguer, Phys. Rev. Lett. 101, 084302 (2008).

${ }^{8}$ J. Christessen, L. Martin-Moreno, and F. J. Garcia-Vidal, Phys. Rev. Lett. 101, 014301 (2008).

${ }^{9}$ X. Wang, J. Appl. Phys. 108, 064903 (2010).

${ }^{10}$ M. Huang, A. Yanik, T. Chang, and H. Altug, Opt. Express 17, 24224 (2009).

${ }^{11}$ A. Taflove, Computational Electrodynamics: The Finite-Difference TimeDomain Method, 2nd ed. (Artech House, Norwood, 2000).

${ }^{12}$ J. Virieux, Geophysics 51(4), 889 (1986).

${ }^{13}$ W. Schaaffs, in Landolt-Boernstein NS5 (Springer-Verlag, Berlin, 1967), p. 18. 